\title{
Management of Hypoxia-induced Disorders in Nephrotic Children
}

\section{Maidannyk $\mathbf{V}^{1}$ and Burlaka $\mathrm{I}^{2 *}$}

Department of Pediatrics No 4, OO Bogomolets National Medical University, Kyiv, Tolstogo Street 10, 01004, Ukraine

\begin{abstract}
Objectives: The objective of this paper was to study the levels of cellular hypoxia, apoptosis controlling factors in relation to the value of kidney function impairment and to evaluate possible protective effects of the treatment with antioxidant agent tocopherol in pediatric patients with nephrotic syndrome.

Background: Hypoxia-related disorders play an important role in irreversible tissue damage via activation of certain secondary processes.

Methods: An examination of renal biopsies of 53 patients (aged 10 to 15 years) with nephrotic syndrome was done. Conventional clinical investigations, immunohistochemistry, immunoblotting were used in this study.

Results: We show that nephrotic patients reveal a high level of marker of cellular hypoxia HIF-1 $\alpha$ and its dependence on the level of kidney function impairment and proteinuria. The progression of the sclerosis as a sign of irreversible kidney damage is accompanied by gradual increase in expression of proapoptotic factor Bax. Applied treatment composes conventional scheme and tocopherol showed an improvement in hypoxia level, proapoptotic factor Bax expression.
\end{abstract}

Conclusion: Hypoxia-induces disorders in nephrotic children presented by disturbances in apoptosis controlling system can be attenuated by the antioxidants application.

Keywords: Treatment; Tocopherol; Nephrotic syndrome; Hif-1 $\alpha$; Bax; Immunostaining

\section{Introduction}

Nephrotic syndrome is kidney disease with proteinuria hypoalbuminemia, and edema. Nephrotic-range proteinuria is 3 grams per day or more. On a single spot urine collection, it is $2 \mathrm{~g}$ of protein per gram of urine creatinine.

There are many specific causes of nephrotic syndrome. These include kidney diseases such as minimal-change nephropathy, focal glomerulosclerosis, and membranous nephropathy. Nephrotic syndrome can also result from systemic diseases that affect other organs in addition to the kidneys, such as diabetes, amyloidosis, and lupus erythematosus. Nephrotic syndrome may affect adults and children, of both sexes and of any race [1].

Main pathomorphological outcomes that apply to kidney damage in nephrotic syndrome are glomerulosclerosis, vascular sclerosis, tubule-interstitial fibrosis [2]. Inflammation plays an important role in the development and progression of chronic proteinuric kidney pathologies and is the primary and persistent violation, which underlies the pathogenesis of others. Renal histology in chronic renal pathologies including nephrotic syndrome is characterized by typical signs of inflammation i.e. infiltration with white blood cells, hyperemia, fibrosis etc. In addition to inflammation, fibrosis has a role in nephrotic syndrome. These disorders are accompanied by activation of the renin-angiotensin-aldosterone system, oxidative stress, endothelial dysfunction and others [1-3]. All mentioned above pathophysiological violations might be accompanied by apoptosis. Apoptosis is programmed cell death that occurs when kidney disease has a place and plays an important role in their physiology. Harmful effects of apoptosis are in fact a source of a large number of kidney cells lost during and/or renal inflammation, scarring, loss of kidney function $[4,5]$ The molecular mechanisms underlying irreversible renal damage in children with nephrotic syndrome depending on apoptosis activation might be a potential therapeutic issue its treatment.
The main tasks in nephrotic syndrome treatment are renal insufficiency compensation, complications prevention and lowering the rate of 6 he disease progression. Basic medicines for nephrotic syndrome treatment are immunoregulative drugs (corticosteroids, immunosuppressive agents), ACEi, Angiotensin receptors blockers. The last two groups provide protective effect through slowing down proteinuria and blood pressure lowering in nephron [6]. However, there are no specific non-toxic drugs regulating apoptosis and apoptosisdependent disorders in this cohort of patients.

\section{Materials and Methods}

\section{Patients}

An examination of renal biopsies of 53 patients (aged 10 to 15 years) with nephrotic syndrome hospitalized in Pediatric Nephrology unit of the Children Clinical Hospital №7 (Kyiv, Ukraine) was done. All patients were treated accordingly to the local protocols. Among all patients 24 (45.28\%) were with hormone-sensitive type of nephrotic syndrome, others- 29 (54.72\%) showed hormone-dependent type of nephrotic syndrome. Complex examination other than conventional methods (inspection, monitoring blood pressure, general and biochemical blood tests, determination of daily proteinuria, urinary sediment study and concentration ability of the kidneys, ultrasound of the abdomen etc.),

*Corresponding author: levgeniia Burlaka, Department of Pediatrics No 4 OO Bogomolets National Medical University, Kyiv, Ukraine, Tolstogo Street 10 01004, Ukraine, Tel: 38-097-432-49-44; E-mail: evgbur1982@gmail.com

Received July 13, 2015; Accepted August 27, 2015; Published August 29, 2015.

Citation: Maidannyk V, Burlaka L (2015) Management of Hypoxia-induced Disorders in Nephrotic Children. J Cytol Histol 6: 362. doi:10.4172/21577099.1000362

Copyright: (c) 2015 Maidannyk V, et al. This is an open-access article distributed under the terms of the Creative Commons Attribution License, which permits unrestricted use, distribution, and reproduction in any medium, provided the original author and source are credited. 
immunohistochemical assessment of apoptosis-dependent glomerular and tubule-interstitial damage were done.

The level of kidney function impairment (stage of Chronic Kidney Disease, CKD) was assessed by the value of glomerular filtration rate (GFR). GFR was calculated by Schwartz formula.

All patients were treated accordingly to local protocols which include corticosteroids (prednisolone), immunosuppressive drugs (CellCept, Leikeran), ACEi, diuretics (furosemide). An antioxidant (Vitamin E - tocopherol) beside mentioned above drugs in dose 100 $\mathrm{mg} /$ day was administered to patients from experimental group for 1 month.

\section{Immunohistochemistry}

Renal tissue (3- $\mu \mathrm{m}$ sections) was deparaffinized and rehydrated prior to processing. Antigen retrieval was carried out by boiling in Citrate Buffer (10 mM Citric Acid, 0.05\% Tween 20, pH 6.0) for 20 min. Sections were treated with Triton X-100 0.3\% (Sigma-Aldrich NV/SA, Bornem, Belgium) in PBS for $20 \mathrm{~min}$. After three PBS washes, sections were incubated with blocking buffer (5\% bovine serum albumin and Triton X-100 0.1\% in PBS) for 1 hour. The rabbit polyclonal anti-Bax antibody (Santa Cruz, CA, USA) was applied at a dilution of 1:50 in $5 \%$ bovine serum albumin (BSA) in PBS overnight at $4^{\circ} \mathrm{C}$. Following three PBS washes, sections were incubated with a secondary Alexa Fluor 488 goat anti-rabbit IgG (1:500) for 1 hour at room temperature. Nuclei were counterstained with DAPI. All samples were stained for an identical length of time and under identical conditions. All recordings for each staining were done during one day using identical gain settings. Sections were mounted in Immu-Mount (Thermo Shandon, Midland, Canada) and observed with Leica TCS SP inverted confocal scanning laser microscope using 25X/0.8NA oil-immersion objective. Image analysis was performed using ImageJ software (NIH Image, Baltimore, MD, USA). Three areas in each section were analyzed.

\section{Immunoblotting for detection of Bax, HIF-1a}

Proteins solubilized in Laemmli sample buffer were resolved in polyacrylamide gels by SDS-PAGE and transferred to a polyvinylidene difluoride membrane. Membranes were then blocked in 5\% nonfat milk in TBS-T (136 mM NaCl, $10 \mathrm{mM}$ Tris, $0.05 \%$ Tween 20 ) and immunoblotted using the Bax and HIF-1a Ab (Cell Signaling Technology, Danvers, MA USA) and actin mouse mAb (BD, Lexington $\mathrm{KY}$, USA) for 1 hour at room temperature. The actin mouse $\mathrm{mAb}$ was used as a loading control. After three washes with TBS-T, the membranes were incubated with secondary anti-rabbit or anti-mouse antibodies labeled with horseradish peroxidase for 1 hour at room temperature. Membranes were washed three times with TBS-T. The protein bands were visualized by chemiluminescent substrate ECL. Quantification of the protein content was done by densitometric analysis.

\section{Statistics}

Statistical analysis was done using the method of variation statistics (STATISTICA 6.0) and nonparametric statistical approaches (MannWhitney test). Results are presented as Mean \pm SEM. $\quad \mathrm{P}<0.05$ was considered as statistically significant.

\section{Results}

We have detected an increased level of marker of cellular hypoxia (HIF-1 a) in blood serum of all patients with nephrotic syndrome as compared to control group. Moreover, dependence between the levels of renal insufficiency (assessed by GFR) and cellular hypoxia has
A
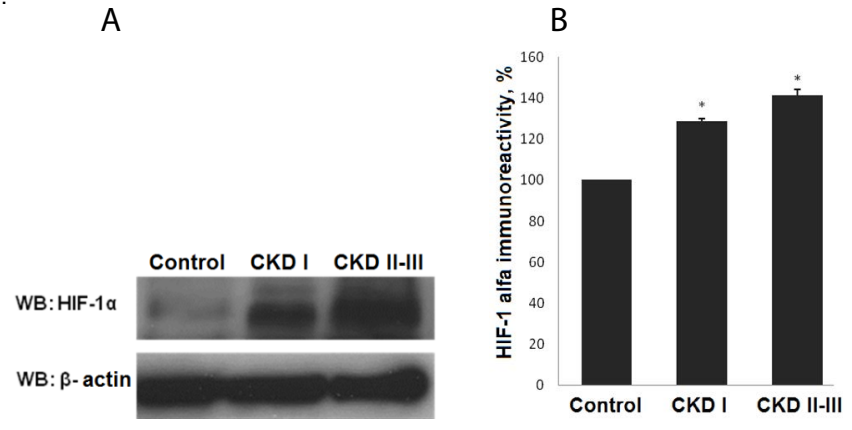

Figure 1: Level of HIF-1 $\alpha$ in plasma of patients with nephrotic syndrome

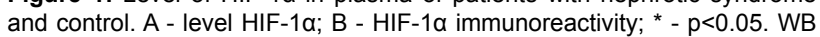
- Western Blotting.

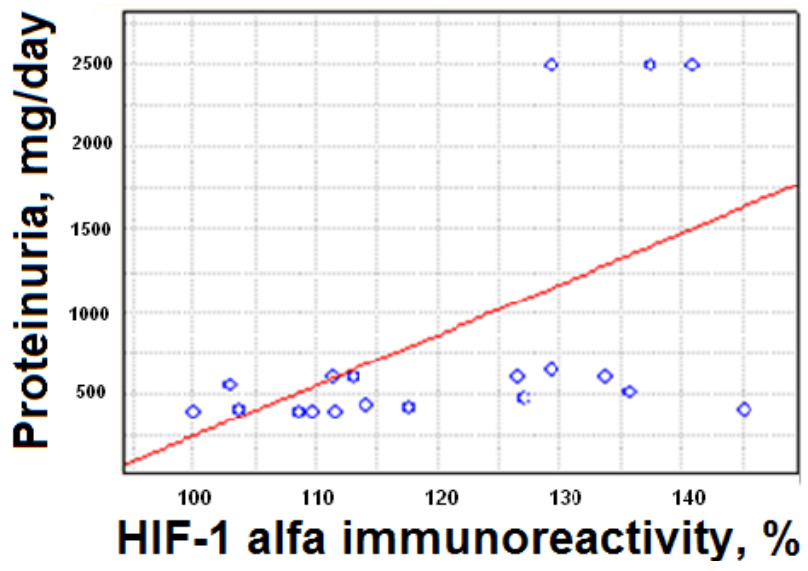

Figure 2: Dependence of HIF-1a levels on daily proteinuria values in children with nephritic syndrome.

been documented. The levels of HIF-1 1 in group with CKD I st. was detected at level over excided the control group value by $28.6 \%(\mathrm{p}<0.01$ compared to control group) and by $41.3 \%$ ( $\mathrm{p}<0.01$ compared to control group) in patients with CKD II-III st. (Figure 1).

Individual analysis of the HIF-1 $\alpha$ levels in serum and correspondent levels of daily proteinuria in patients with nephritic syndrome identified a direct positive correlation $(\mathrm{r}=0.61, \mathrm{p}<0.01)$ (Figure 2$)$.

These data indicate the dependence of chronic hypoxia on the degree of damage to the kidney filtration barrier and is a proof of the direct influence of hypoxia and CKD progression.

\section{Expression of proapoptotic factor Bax in kidney tissue of patients with nephrotic syndrome}

We have analyzed the levels of expression and localization of proapoptotic factor Bax in patients with morphological variant of nephrotic syndrome - focal segmental glomerulosclerosis. Stages of FSGS were determined by level of glomerular sclerotic area. Level of sclerosis corresponding to $\leq 25 \%$ of the glomerular area was assumed as I stage of FSGS, II stage of FSGS - 25-50\%, III stage - 50-75\% and IV stage - 75-100\%. Analysis of Bax expression in kidney biopsies from children with focal segmental glomerulosclerosis show the presence of high level of Bax expression in both glomerular and tubule-interstitial segments. Higher level of immune signal was recorded in glomeruli as compared to tubule-interstitial segment in FSGS I-II stages (43.57 \pm 0.88 a.u. vs $24.9 \pm 0.41$ a.u., $\mathrm{p}<0.01)$. When complete glomerular sclerosis 
Citation: Maidannyk V, Burlaka L (2015) Management of Hypoxia-induced Disorders in Nephrotic Children. J Cytol Histol 6: 362. doi:10.4172/21577099.1000362

presents a high level of Bax was documented in the surrounding tubuleinterstitial segment ( $13.7 \pm 0.42$ a.u. vs $22.5 \pm 0.65$ a.u., $\mathrm{p}<0.01)$ (Figure 3 ).

\section{Therapeutic effects on hypoxia-induced damages in patients with nephrotic syndrome}

To evaluate the effectiveness of treatment of children with nephritic

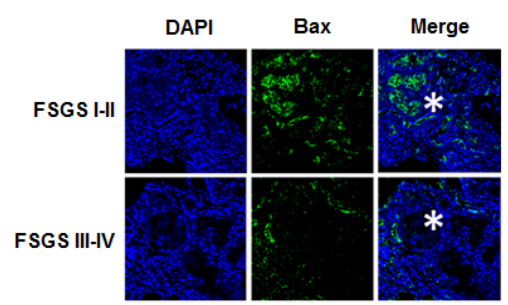

A

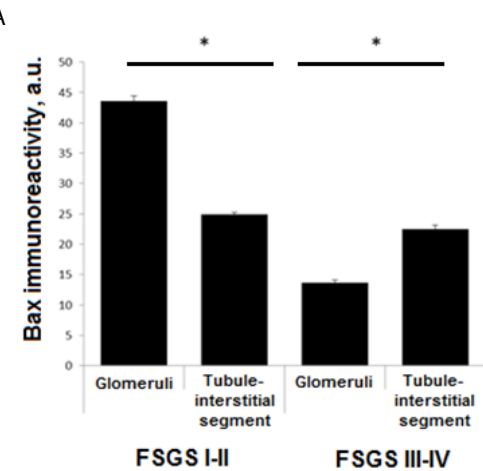

Figure 3: Topical characteristic of the Bax expression in different stages of FSGS. DAPI - visualization of nuclei; Bax - Bax immune signal in kidney tissue. ${ }^{*}$ - glomeruli, ${ }^{*} p<0.05$.
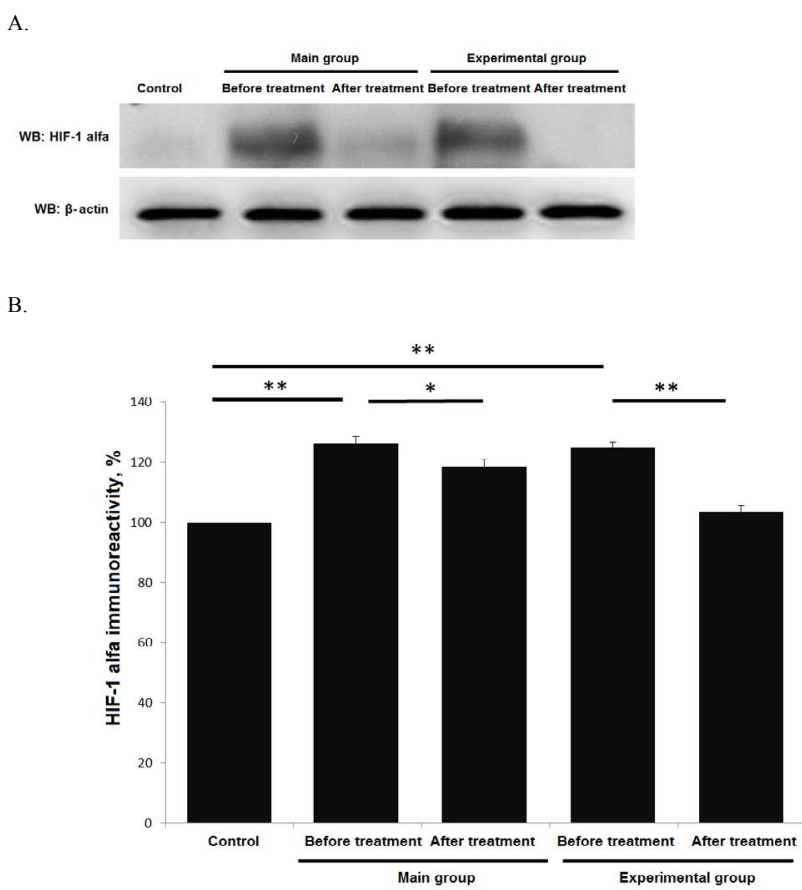

Figure 4: Levels of hypoxia HIF-1a in children with nephrotic

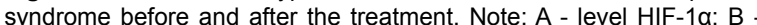
immunoreactivity; ${ }^{*}-p<0.01,{ }^{* *}-p<0.001$.

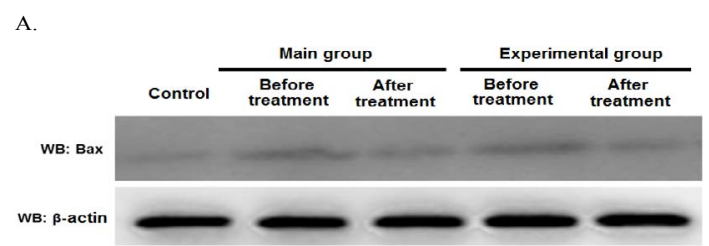

B.

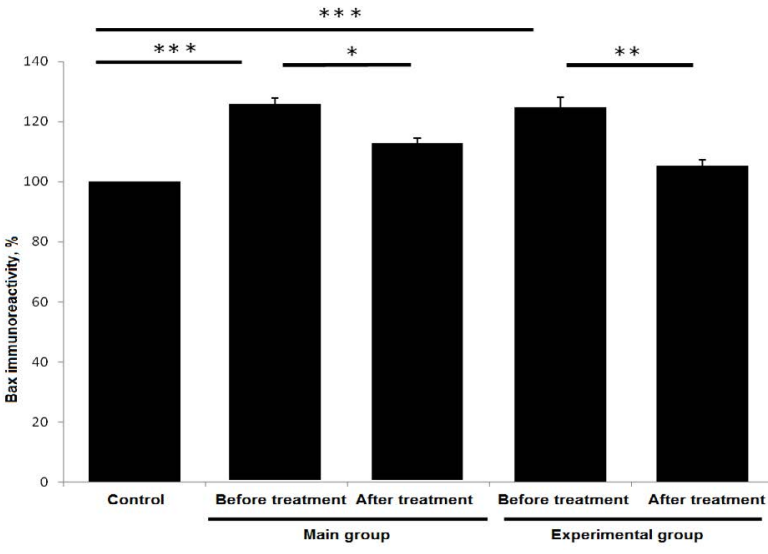

Figure 5: Levels of proapoptotic factor Bax in children with nephritic syndrome before and after the treatment. A - level of Bax; B immunoreactivity; ${ }^{*}-p<0.05,{ }^{* *}-p<0.01,{ }^{* * *}-p<0.001$

syndrome all patients were randomized and divided into three groups. The first group (main group) were children with nephrotic syndrome who received conventional basic therapy. The second (experimental group) were children with nephrotic syndrome who received conventional basic therapy and tocopherol. The third group (control) included 45 healthy children matched by age and sex.

After a course of therapy all children were investigated for serum level marker of chronic hypoxia HIF-1a. In children from from main group the level of HIF-1 $\alpha$ exceeded indicator of the control group by $26.23 \pm 2.2 \%(\mathrm{p}<0.001)$. After a course of treatment the level HIF- $1 \alpha$ in this group exceeded control rate by $18.56 \pm 2.49 \%$ ( $p<0.01$ in comparison to the level before treatment). Children from the experimental group showed a level of HIF-1 $\alpha$ exceeded the control group by $24.9 \pm 1.76 \%$ ( $\mathrm{p}<0.001)$. After the course of treatment HIF-1a was decreased almost to the level of control group (Figure 4).

After the course of therapy all examined children were investigated for serum levels of proapoptotic factor Bax. In children included to the main group Bax concentration was increased up to $124.7 \pm 3.45 \%$ (p $<0.001$ ) as compared to control. Value of control control group was taken as $100 \%$. After a course of treatment Bax value was decreased to $112.3 \pm 1.6 \%(\mathrm{p}<0.05)$ as compared to level before treatment. Children from experimental group exhibited Bax before treatment at level $125.5 \pm 1.9 \%(\mathrm{p}<0.001)$ as compared to control group. After a course of treatment Bax was down regulated to $105.2 \pm 2.02 \%(\mathrm{p}<0.01$ in comparison to the level before treatment) (Figure 5).

\section{Discussion}

It is accepted that proteinuria is a hallmark of glomerular disease, and the magnitude of proteinuria is an adverse prognostic factor in 
varied nephropathies including nephrotic syndrome. There is evidence that proteinuria is a mechanism of kidney disease progression. A reduction in proteinuria is associated with a slower decline in GFR. Enhanced albuminuria leads to the secondary pathologycal prosecces inflammation, hypoxia, fibrosis [7]. Ischemia as a result of peritubular capillary loss or hypoperfusion is also considered a major factor for the progression of tubulointerstitial damage, which is closely associated with impairment of renal function. Renal tissue hypoxia induces profibrogenic responses and tubulointerstitial injury, which includes degeneration, dedifferentiation, cell death.

The hypoxia-inducible factors (HIFs) comprise a family of oxygen-sensitive basic helix-loop-helix proteins that control cellular transcriptional response to hypoxia [8]. Hypoxiainducible factor 1-alpha, also known as HIF-1-alpha, is a subunit of a heterodimeric transcription factor hypoxia-inducible factor 1 (HIF-1) that is encoded by the HIF1A gene. It is a basic helix-loophelixPAS domain containing protein, and is considered as the master transcriptional regulator of cellular and developmental response to hypoxia. The dysregulation and overexpression of HIF1A by hypoxia have been heavily implicated in a number of pathophysiologies, specifically in areas of vascularization and angiogenesis, energy metabolism, cell survival. It was shown that HIF signaling could potentially promote the development of fibrosis particularly due its role in inflammation $[8,9]$.

Pathogenesis of the nephrotic syndrome deals with inflammation leading to progression and development of the irreversible tissue damages. Hypoxia is an important link in inflammation development [10]. A mechanism by which HIF may impact the pathogenesis of kidney damage is through the regulation of inflammatory responses. Microenvironmental changes, such as hypoxia, strongly impact inflammatory cell recruitment and function. Moreover, hypoxia has been shown to induce apoptosis, where HIF-1 plays a complex role. It has also been demonstrated that the expression of HIF-1a significantly correlated with apoptosis and the proapoptotic factors, such as caspase-3, Fas, and Fas ligand. This finding has been shown on in vitro models [11].

Here we show that HIF-1 $\alpha$ and Bax were overexpressed in children with nephritic syndrome meaning that chronic hypoxia is a factor predisposing disturbances in system controlling apoptosis in this cohort of patients. Our data demonstrate that there is dependence between the level of hypoxia, Bax overexpression and the stage of CKD. We show that progression of glomerulosclerosis in children with nephrotic syndrome is accompanied by increased activity of proapoptotic factor. Revealed dependence of topology of Bax levels on FSGS degree indicates that development of glomerular and tubule-interstitial disorders under the influence of proteinuria occurs in specific range.

Tocopherol is a fat-soluble antioxidant that is able to intercept free radicals in the plasma membrane, which helps to prevent the oxidative damage to lipids. It is known that patients with CKD have reduced levels of tocopherol in plasma, which is a prerequisite for this use in therapies [12]. Previous studies have shown that administration of tocopherol in patients with CKD helped to reduce the risk of cardiovascular complications, increased activity of endogenous antioxidant systems GPX, catalase [13]. It has been shown that treatment with an antioxidant lowered the hypoxia-induced cell damage as well as the elevation of HIF-1alpha in vitro [14]. Here we demonstrate that tocopherol administration has a potent effect on hypoxia-induced apoptosis development in children with nephrotic syndrome and subsequent restoration of the proapoptotic factor Bax activation. We speculate that this therapeutical intervention may be a new non-toxic approach in fibrosis prevention in chronic albuminuric kidney pathologies.

Further study of the molecular mechanisms which provides kidney protection in nephrotic patients upon the treatment with antioxidants in addition to the conventional therapy will be done.

\section{Acknowledgements}

This work has been done with support from nephrology unit of Children Clinical Hospital №7 (Kyiv, Ukraine) and by the grant 0105 U 003769.

\section{References}

1. Eddy AA, Symons JM (2003) Nephrotic syndrome in childhood. Lancet 362 629-639.

2. Hemmelgarn BR, Manns BJ, Lloyd A, James MT, Klarenbach S, et al. (2010) Relation between kidney function, proteinuria, and adverse outcomes. JAMA 303: 423-429.

3. Favaloro B, Allocati N, Graziano V, Di llio C, De Laurenzi V (2012) Role of apoptosis in disease. Aging (Albany NY) 4: 330-349.

4. Christman JW, Blackwell TS, Juurlink BHJ (2000) Redox Regulation of Nuclear Factor Kappa B: Therapeutic Potential for Attenuating Inflammatory Responses. Brain Pathology 10: 153-162.

5. Abbate M, Zoja C, Remuzzi G (2006) How does proteinuria cause progressive renal damage? J Am Soc Nephrol 17: 2974-2984.

6. KDIGO (2012) Clinical Practice Guideline for the Evaluation and Management of Chronic Kidney Disease. Kidney Int Suppl 3: 5.

7. Gordillo R, Spitzer A (2009) The nephrotic syndrome. Pediatr Rev 30: 94-104.

8. Bento CF, Pereira P (2011) Regulation of hypoxia-inducible factor 1 and the loss of the cellular response to hypoxia in diabetes. Diabetologia 54: 1946-1956.

9. Eltzschig HK, Carmeliet P (2011) Hypoxia and inflammation. N Engl J Med 364: 656-665.

10. Du R, Xia L, Ning X, Liu L, Sun W, et al. (2014) Hypoxia-induced Bmi1 promotes renal tubular epithelial cell-mesenchymal transition and renal fibrosis via PI3K/ Akt signal. Mol Biol Cell 25: 2650-2659.

11. Volm M, Koomägi R (2000) Hypoxia-inducible factor (HIF-1) and its relationship to apoptosis and proliferation in lung cancer. Anticancer Res 20: 1527-1533.

12. Bin $Q, H u X$, Cao $Y$, Gao $F$ (2011) The role of vitamin $E$ (tocopherol) supplementation in the prevention of stroke. A meta-analysis of 13 randomised controlled trials. Thromb Haemost 105: 579-585.

13. Young JF, Rosenvold K, Stagsted J, Nielsen JH, Andersen HJ (2005) Significance of vitamin $\mathrm{E}$ supplementation, dietary content of polyunsaturated fatty acids, and preslaughter stress on oxidative status in pig as reflected in cell integrity and antioxidative enzyme activities in porcine muscle. J Agric Food Chem 53: 745-749.

14. Magalhães J, Ferreira R, Neuparth MJ, Oliveira PJ, Marques F, et al. (2007) Vitamin $E$ prevents hypobaric hypoxia-induced mitochondrial dysfunction in skeletal muscle. Clin Sci (Lond) 113: 459-466. 